\title{
Immuno Correction of Genital Herpes of Viral Infection Moderate and Severe Course Betaleikin
}

\author{
L.K. Smailova ${ }^{1,3 *}$, A.M. Kurmanova ${ }^{1}$, G.M. Kurmanova ${ }^{2}$, \\ R.R. Razumova ${ }^{3}$ and A.A. Eszhanova ${ }^{3}$
}
${ }^{1}$ Scientific Centre of Obstetrics, Gynecology and Perinatology of Ministry of Public health and Social Development of the Republic of Kazakhstan, Almaty
${ }^{2}$ Kazakh National Medicinal University, Republic of Kazakhstan, Almaty
${ }^{3}$ JSC, Astana Medical University, Republic of Kazakhstan, Astana

*Corresponding author

A B S T R A C T

\begin{tabular}{|l|}
\hline K e y w or d s \\
Genital herpes, \\
Viral infection, \\
Immuno \\
Correction
\end{tabular}

\section{Introduction}

In the structure of sexually transmitted infections, the infection caused by herpes simplex virus (HSV-infection) is one of most prevailing and socially important diseases
(Kinghorn, 1994; Jansen et al., 2015). Recurrent course of herpes genitalisis observed in $50-75 \%$ infected individuals (Grenebyuk, 1993; Zudin and Kissina, 1995). Numerous studies demonstrated existing relationship between $\mathrm{HSV}$-infection and 
transmission of HIV-infection as well as development of oncologic pathology of genitals in women (Marchenko, 2000).

Immunity formation in herpes virus infections is complex and multicomponent process, during which the cell cooperation can be disturbed at different stages. Thus, for example, activity of macrophages, viral infection nature and delivery of virus-specific antigen to B-lymphocytes at inductive stage of herpes determine body resistance to this infection. At the other side, specific cell immunity, mediated by T-lymphocytes, has decisive influence on herpetic infection. Herpetic infection, like other chronic diseases with virus persistence, causes development of immune suppression due to insufficiency of different immune system sections and its inability to eliminate virus from the body (Kurmanova et al., 2014). At the time, it is proven that viral infections are accompanied by disturbed immune response regulation on the basis of interleukins (IL) system disturbances. These disturbances can have few development mechanisms. In some cases, ability of immune competent cells to IL synthesis will be inhibited, in other cases, target cells reaction to IL changes. In particular, it was demonstrated that infection caused by type II herpes simplex virus inhibits interleukin-1 production (Simbirtsev, 2002). But a lot is unknown in the cell immunity mechanism, e.g., what promotes or, on the contrary, interrupts infection recurrences (Isakov et al., 2004).

Despite the large arsenal of anti-herpetic drugs, including preparations of interferons and their inductors, immune stimulating drugs and anti-herpetic vaccines, herpes relapses are quite frequent (Aspetov, 2006). It is known that IL-1 $\square$ stimulates both non-specific resistance mechanisms related mostly to activation of neutrophil leukocytes functional activity, and specific immune response, increases NK-cells activity and induces mediated endogenous interferon synthesis (Simbirtsev, 2002). Therefore, complex study of some features of immunological shifts in subpopulation lymphocytes composition, cytokine system and non-specific defense factors at recurrent herpes reveals possibilities of new approaches to immune correction with the interleukin-1 preparation (Betaleukin).

\section{Study goal}

To examine clinical and immunological effectiveness of recombinant IL-1 $\square$ in severe herpetic infection.

\section{Materials and Methods}

To perform assigned tasks, ambulatory cards of 108 women with moderate and severe HSV infection were analyzed. Average age of study women was $32.8 \pm 5.7$ years. Women with herpetic infection were distributed by age as follows: $17-24$ years - 21 (19.4\%), $25-29$ years - $32(29.6 \%), 30-39$ years -42 $(38.9 \%), 40-44$ years $-10(9.3 \%)$ and 45 years and older $-3(2.8 \%)$.

Diagnosis of HSV infection was made on the basis of clinical symptoms (skin rashes, intoxication symptoms and fever) and results of HSV detection in immunofluorescence reaction and specific antibodies in immuneenzyme analysis.

Study of clinical and immunological effectiveness for different therapy schemas in 60 recurrent herpes patients was performed:

$1^{\text {st }}$ group (15 patients) - prescription of acyclovir $200 \mathrm{mg} 5$ times a day during relapse period,

$2^{\text {nd }}$ group (30 patients) - interleukin-1 $\beta$ (Betaleukin, State scientific-research institute of very pure bioproducts / Sankt-Petersburg, 
RF) $500 \mathrm{ng}$ was applied after day 5 subcutaneously in combination with acyclovir during relapse period,

$3^{\text {rd }}$ group (15 patients) - interleukin- $1 \beta$ during relapse period.

Drug effectiveness evaluation was performed based on standard form of numeric evaluation of complaints and symptoms in patients with manifested form of genital herpes virus infection.

Clinical examination of women included collection of epidemiologic and obstetricgynecologic anamnesis, general examination, special gynecological examination, bacterioscopic examination.

Urogenital infections diagnosis was determined using antigens detection in RIF ("Bioservice"/Moscow, RF) and specific antibodies in IFA ("Vector-Best"/Koltsovo, $\mathrm{RF}$ ).

Subpopulation lymphocytes analysis was performed on flow cytofluorimeter "Becton Dickenson"/USA using FacsCalibur program with a method modified by NII Immunology $\mathrm{MoH}$ RF with application of monoclonal antibodies panel to superficial antigens of lymphocytes (NPO "Sorbent"/Moscow/RF). Ripe lymphocytes CD3+, T-helpers CD4+, cytotoxic lymphocytes CD8+, natural killers CD16+, CD56+ and B-lymphocytes CD20+, activation markers CD25+, CD95+, HLA$\mathrm{DR}+$ and adhesion markers CD11b+ were determined.

Phagocytic system activity evaluation in phagocytosis reaction with latex and NST-test in spontaneous and stimulated LPS variants.

Cytokine profile of solid-phase IFA with a set of test systems Victor-Best (Novosibirsk, RF) and "Cytokine" (SPb, RF) IL1 $\beta$, IL4, INF $\square$,
FNO $\square \quad$ on IFA-reader "AnthosLabtec Instruments Zenuth".

Statistical treatment of obtained study results was performed using Student's t-test with $\mathrm{P}<$ 0.05 probability.

\section{Results and Discussion}

We analyzed ambulatory cards of 108 women 20 to 50 years of age (mean age $32.8 \pm 5.7$ years) having moderate and severe HSV infections, observed in dermatovenerologic and antenatal clinics of Almaty.

Study results analysis has shown that clinical symptoms manifestation varied depending on disease remoteness and herpetic infection severity. In overwhelming majority of patients $(55.5 \%)$, frequency of herpetic infection relapses was 3 to 5 times (on average $5.6 \pm 3.4$ times) per year. Relapse duration varied 6 to 14 days and was $10.4 \pm 4.2$ days on average.

All patients had certain herpetic infection manifestations. In almost all cases, patients complaints about periodic rashes on skin and mucosa, accompanied by tenderness $(83.3 \%)$, itch $(90.7 \%)$ and burning (100\%). In $100 \%$ of cases, intoxication symptoms were observed manifested as weakness, chill, headache. $92.6 \%$ female patients had fever. Skin rashes were accompanied by hyperemia in $83.3 \%$ women, edema - in $46.3 \%$. In $92.6 \%$ women, rashes were vesicular, $50.9 \%$ of them transformed into erosions. Regional lymphadenitis was noted in $41.7 \%$ women. In 8 patients $(7.4 \%)$, pains in joints and muscles were observed.

Clinical symptoms of herpetic infection had different intensity. Based on that, evaluation of each clinical symptoms was performed using the numerical score. Average score of recurrent herpes clinical manifestation in women was $17.6+0.2$. 
In recurrent herpes, possible cause of herpetic infection reactivation is the presence of a mixed infection. In 12 (11.2\%) women, only HSV was detected, remaining 96 (88.8\%) had combinations with other urogenital infections. Most often were combinations with CMV, mycoplasma, trichomonas $(33.3 \%)$, chlamydia $(30.6 \%)$. At that, in 50 (46.3\%), concomitant presence of three-four microorganisms in urogenital tract was detected.

According to (Kozlova and Pukhner, 2003), in the case of mixed infection, a possibility of synergic action of agents on inflammatory process in whole cannot be excluded, at that, the formation of specific antibodies will be inhibited. Thus, from 108 women with herpetic infection, HSV antigen was detected in RIF of 75 (69.4\%) examined, in PCR - in $12(11.2 \%)$. At that, in IFA, specific antigens to HSV were registered in 24 (22.2\%) patients.

Therefore, at moderate and severe HSV, clinical manifestations intensity depended of disease remoteness and presence of mixed infections, promoting secondary immune deficient conditions development, supporting prolonged persistence.

Condition of human cell immunity substantially determines the character of herpetic infection course, frequency and intensity of relapses (Table 1).

It was established that in the group of women with moderate and severe HSV-infection, relative CD3+lymphocytes content was significantly decreased $(\mathrm{P}<0.001)$. Also reliable decrease of relative $\mathrm{CD} 3+-$ lymphocytes content was noted. Decreased immune regulatory index $(1.0 \pm 0.04)$ due to decreased relative CD4+lymphocytes content was observed. CD8+-lymphocytes content was not different from control data. At the same time, relative B-lymphocytes content was reliably $(\mathrm{P}<0.05)$ increased. Reliable ( $\mathrm{P}$ $<0.05)$ increase of relative natural killers content was registered, due to CD16+cells.

Analysis of activation markers expression has demonstrated that relative CD25+lymphocytes content were reliably $(\mathrm{P}<0.01)$ increased comparing to analogous control group value. Relative content of cells, ready for apoptosis (CD95+), was reliably $(\mathrm{P}<0.01)$ increased. Reliably increased was content of lymphocytes, expressing adhesion receptors $(\mathrm{CD} 11 \mathrm{~b}+)$. Expression of early activation marker HLA-DR+ on surface of lymphocytes had no statistical differences from control due to large dispersion of individual values.

During genital herpes, viral particles infecting urogenital tract mucosa, meet mechanical protective factors, which are a barrier on the way of virus invasion, and include mucosa itself, normal bacterial flora and glycocalix. Complement has neutralizing effect on the virus before its attachment to target cells. IgM, secreting by CD5+ B1-lymphocytes, very effectively activate the complement system, opsonize virions or infected cells, promote their lysis or phagocytosis. Macrophages, in their part, absorb virions and cells damaged by the virus, but at the same time, they remain immune to herpetic infection. These cells elements become an important source of inflammation chemokines and cytokines. Macrophages are also the main source of nitric oxide (NO) - compound having significant antiviral activity. In peripheral blood during moderate to severe HSV-infection, reliable decrease of spontaneous $(\mathrm{P}<0.05)$ and induced LPS $(\mathrm{P}<0.01)$ latex phagocytosis was found. Functional activity of peripheral blood microphages in spontaneous NST-test values has no reliable difference comparing to control. In LPS stimulation, the number of NST neutrophils with positive response was lower than in healthy subjects, but there were no reliable differences. 


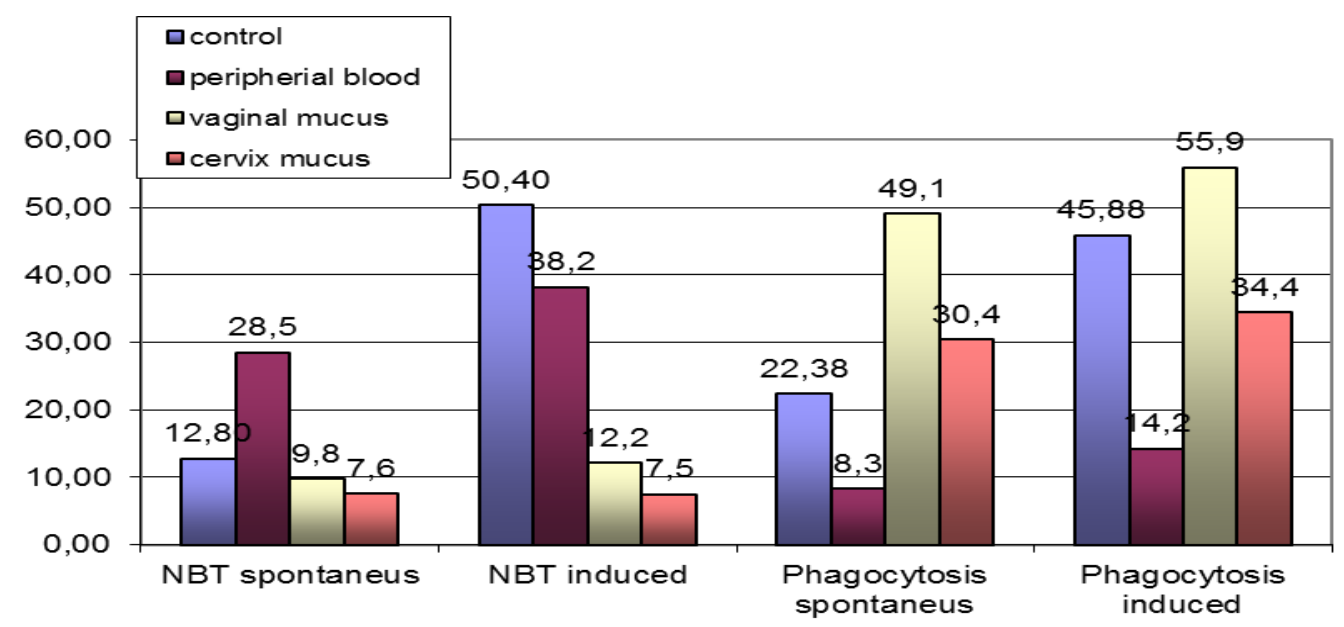

Picture 1. Phagocytosis and NBT-test indicators, \%

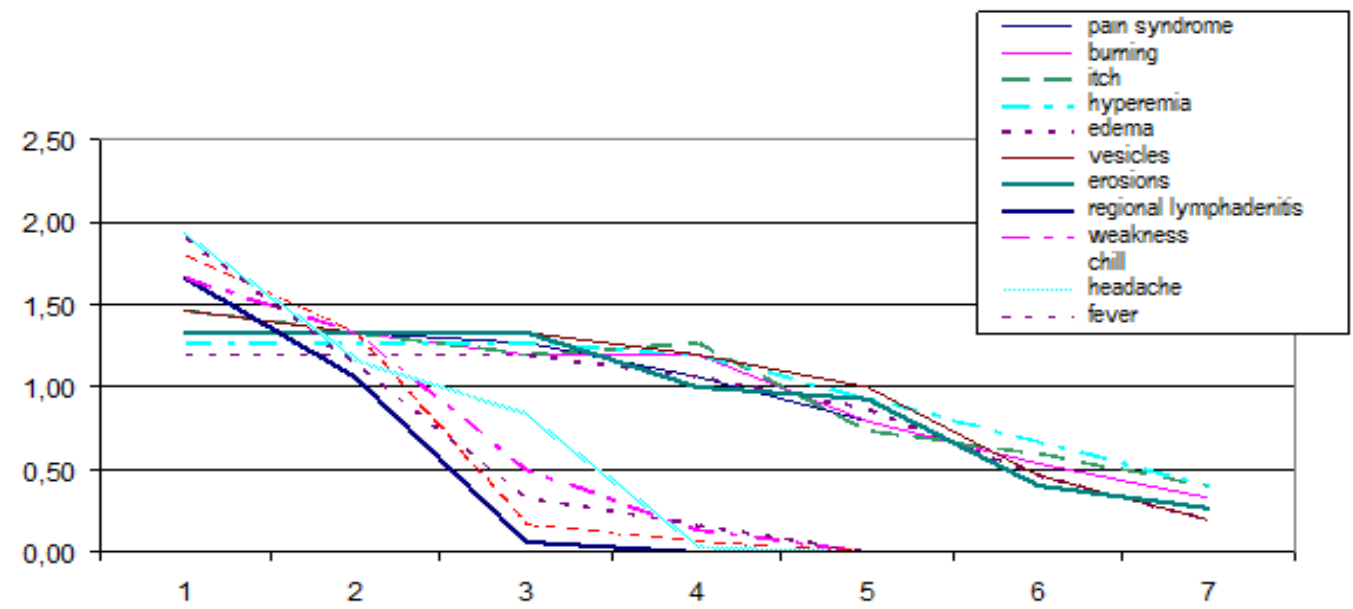

Picture 4 - Dynamics of RH clinical manifestafons on the background of betaleukin with acyclovir (in points)

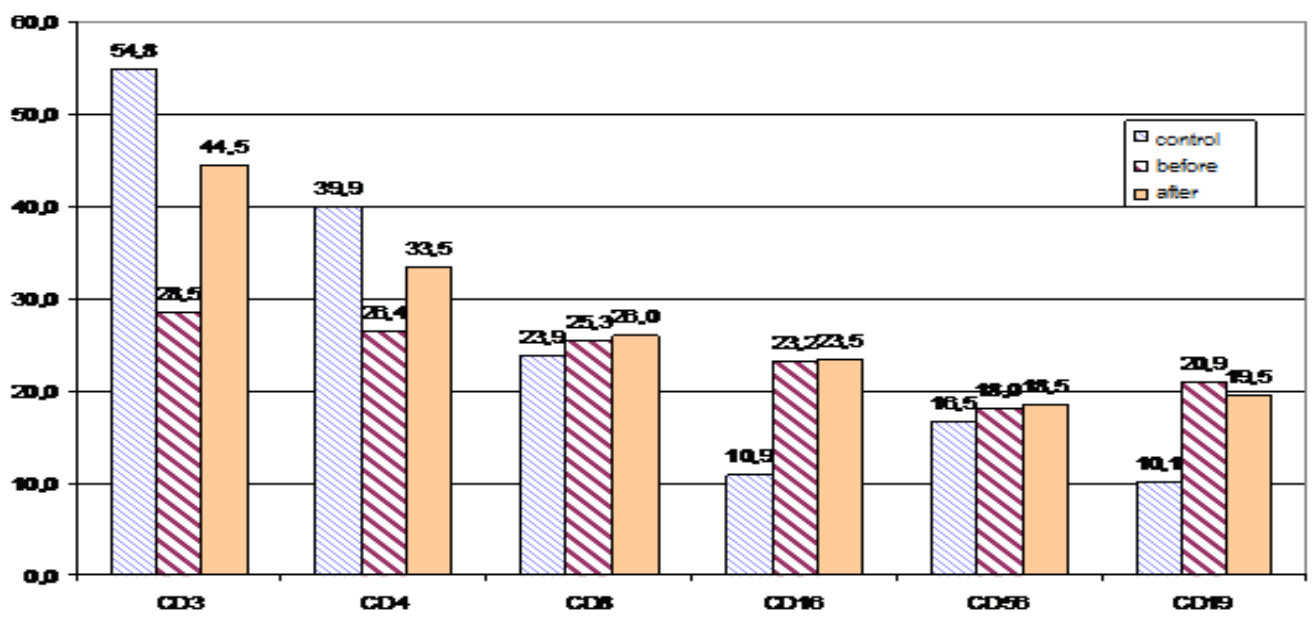

Picture 5 - Dynamics of lymphocytes population composition data on the background of betaleukin, \% 


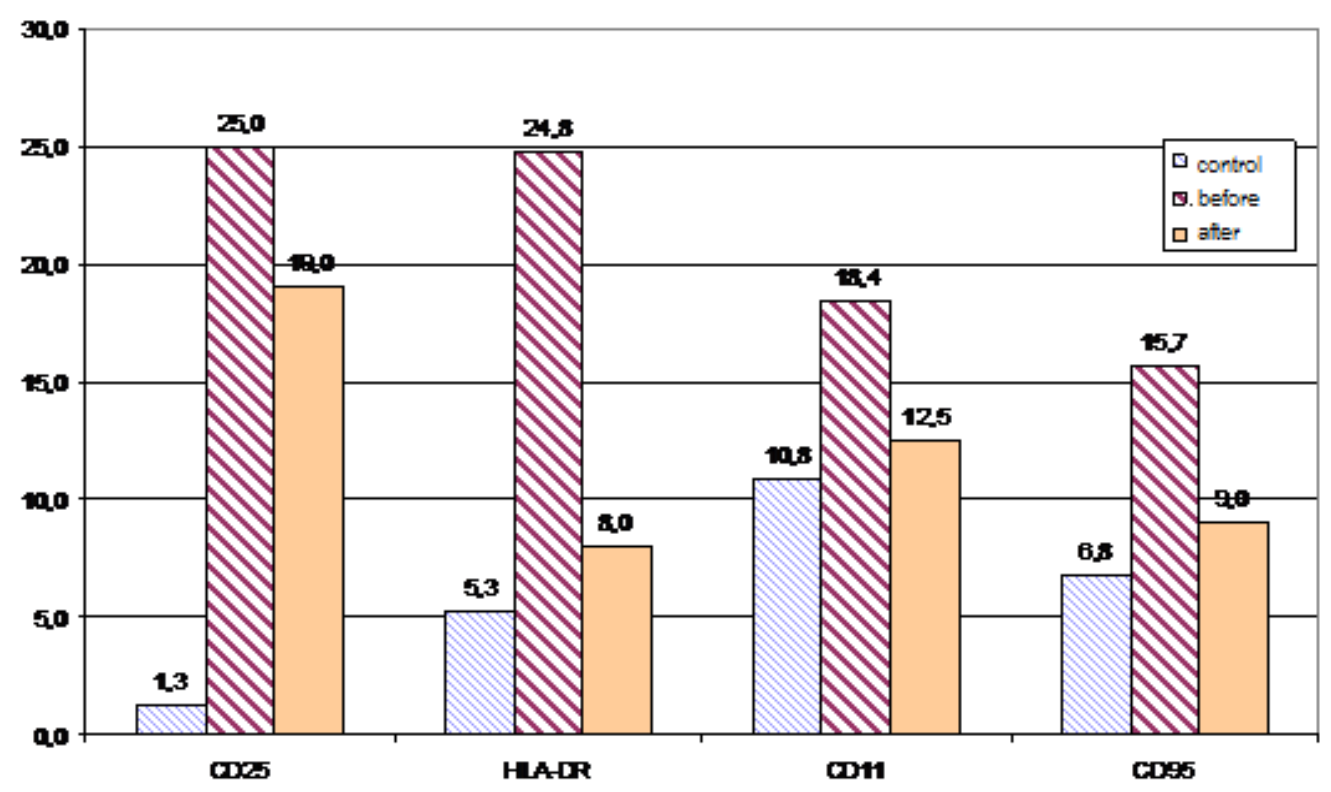

Picture 6 - Dynamics of activation markers data on the background of betaleukin, \%

Table.1 Relative content of lymphocytes subpopulations in women with inflammatory genital diseases on the background of herpetic infection $(\mathrm{M} \pm \mathrm{m})$

\begin{tabular}{|l|c|c|}
\hline \multicolumn{1}{|c|}{ Index, $\%$} & Control, $\mathrm{n}=30$ & Main group, $\mathrm{n}=30$ \\
\hline Lymphocytes & $37.0 \pm 7.3$ & $43.2 \pm 5.6$ \\
\hline CD3+ & $54.8 \pm 3.2$ & $28.5 \pm 4.0^{* * *}$ \\
\hline CD4+ & $39.9 \pm 3.3$ & $26.4 \pm 3.52^{*}$ \\
\hline CD8+ & $23.9 \pm 2.8$ & $25.3 \pm 3.24$ \\
\hline CD16+ & $10.9 \pm 2.6$ & $23.2 \pm 3.4^{*}$ \\
\hline CD56+ & $16.5 \pm 1.0$ & $18.0 \pm 5.4$ \\
\hline CD20+ & $10.1 \pm 3.0$ & $20.9 \pm 3.48^{*}$ \\
\hline CD11b+ & $10.8 \pm 2.2$ & $18.4 \pm 2.1 *$ \\
\hline CD25+ & $1.3 \pm 0.1$ & $25.0 \pm 7.0 * *$ \\
\hline CD95+ & $6.8 \pm 1.1$ & $15.7 \pm 2.3 * *$ \\
\hline HLA-DR+ & $5.3 \pm 0.6$ & $24.8 \pm 12.8$ \\
\hline Note: $*$ - differences confidence $\mathrm{P}<0.05$ comparing to control \\
$* *-\mathrm{P}<0.01$ & & \\
$* * * \mathrm{P}<0.001$ & &
\end{tabular}

In vaginal and cervical mucus, indices of spontaneous and induced LPS latex phagocytosis have no reliable difference comparing to corresponding control values. Digestive activity of vaginal secretion neutrophils, based on NST-test data, was reliably $(\mathrm{P}<0.05)$ decreased both in spontaneous and stimulated variants. Digestive activity of cervical secretion neutrophils was inhibited in even greater extent, both in spontaneous $(\mathrm{P}<0.05)$ and in stimulated $(\mathrm{P}<0.01)$ variants (Picture 1$)$. 
In the examination of peripheral blood cytokine state (IL1 $\beta$, FHO $\square$, INF $\square$ and IL4), average cytokine values varied in wide ranges. At individual values analysis, both increased and low values were registered. In women with HSV-infection, increased content of Tx1-profile INF $\gamma$ cytokines was observed in $43.3 \%$ of examined women, at that, adequately high (4fold) content was registered in the half of them (16.7\%). Increased FNO $\square$ content was less typical in this group, and registered in only $6.7 \%$ female patients, high FNO $\square$ production was not observed in any patient. Increased IL$1 \beta$ production was in $10 \%$ of women, but high level of IL-1 $\beta$ production was noted in only $3.3 \%$.

Increased IL-4 production, reflecting Tx- 2 type of immune response, was not observed. In cytokine profile analysis, there is an impression that during the HSV-infection, adequately high INF $\square$ production is observed in only $1 / 6$ of patients, and high IL-1 $\beta$ production - in single female patients. Thus, in women with herpetic infection, deficiency of anti-inflammatory cytokines is observed, both of Tx1- and Tx-2 profile.

Complex examination of subpopulation lymphocytes composition, non-specific immunological reactivity and cytokine state demonstrated that in moderate to severe HSVinfection, pronounced T-cell section depression is noted, manifested in reliable decrease of relative $\mathrm{CD} 3+-, \mathrm{CD} 4+-$ lymphocytes content, whereas relative content of B-lymphocytes and natural killers of CD16+-cells and lymphocytes, expressing adhesion receptors $\mathrm{CD} 11 \mathrm{~b}+$, and early and late activation markers. compensatory was increased; in phagocytic system - on the systemic level, catching ability of microphages was inhibited and digestive ability is satisfactory, on the local level, on the contrary, phagocytic activity of neutrophils is satisfactory, and digestive function is inhibited, at that, metabolic activity inhibition is in greater extent typical for cervical secretion; deficiency of both Tx1- and Tx2-profile cytokines will be observed.
Clinical activity study for acyclovir $200 \mathrm{mg} 5$ times a day during moderate to severe HSVinfection has shown that at the first day of therapy, decrease of local manifestations was noted only by $46.7 \%$ patients with moderate disease course. In some patients, eruptions of new elements were observed. Tendency to their decrease at treatment days 4 to 5 was observed in $60 \%$ female patients. At day 7, existing components decreased in their size, and in most cases were represented by small erosions or crusts. Intoxication symptoms intensity decreased at day 4. In following, regress of herpes clinical manifestations occurred at treatment day 6 to 7 . Complete epithelization of elements and disappearance of systemic manifestations were observed only at days 11 to 14. Thus, at acyclovir application, clinical recovery occurred at days 11 to 14 after treatment start. Performed treatment has no significant effect on relapse terms. In all patients, relapses occurred after $1.5-2$ months. Acyclovir therapy also had no significant effect on immune state indices.

Study of interleukin-1ßeffectiveness in $500 \mathrm{ng}$ dose every second day during five days in combination with acyclovir $200 \mathrm{mg} 5$ times a day at moderate to severe HSV infection has demonstrated, that in almost all patients the regression of clinical manifestations of herpes occurred at treatment day 2 to 3 . At day 4, all local manifestations with epithelization of elements disappeared completely. Performed treatment led to remission duration increased more than 2-fold. When before the treatment in 18 patients with severe course, remission duration was 1.2 - 1.5 months, after the treatment with interleukin-1 $\beta$ with acyclovir, in 17 patients duration increased to 12 months, and in 1 female patient remission duration has not changed. In 12 patients with moderate course, remission duration was $2-3$ months, after the treatment with interleukin-1 $\beta$ with acyclovir, in 3 patients remission continued 18 months, and 9 had no relapses at all during 2 years (Picture 2).

Therefore, on the background of therapy with interleukin-1 $\beta$ with acyclovir, significant 
improvement was noted by $96.6 \%$ (29/30) patients.

When our interleukin-1 $\beta$ effectiveness evaluation results were compared with treatment results when IIBL was prescribed in combination with herpetic vaccine (Nygmanova, 2005), with which reduction of topical and general herpes virus infection symptoms occurred at days 1 to 4 , and with relapse-free period up to 1.5 years and more, interleukin-1ßapplication allowed to reduce clinical manifestation in same time, but remission duration increased to 2 years.

Immuno stimulating action of IL-1 $\square$ is the most important feature of this cytokine, and forms the basis of whole complex of resulting biological effects, directed to formation of body defensive reactions. IL-1 $\square$ stimulates both non-specific resistance mechanisms, related mostly to activation of functional activity of neutrophilic leukocytes, and specific immune response.

After the treatment course with interleukin$1 \beta$ with acyclovir, patients had normalization of initially decreased CD3+, CD4+ content, CD8+ lymphocytes content increased, CD16+, CD56+, CD20+ lymphocytes content remained at same high level (Picture 5).

Analysis of dynamics of activation markers expression has demonstrated that relative and absolute CD25+ lymphocytes content remained on reliably $(\mathrm{P}<0.05)$ high level, and $\mathrm{CD} 95+$ cells content had a tendency to reducing. After the treatment, values of spontaneous and induced LPS NST-test increased significantly (Picture 6).

Follow-up of patients during one year had shown that the main group has no herpes relapses, and the level of specific antibodies in IFA even increased compared to initial data, and decreased only 6 months after the treatment course. In the control group, herpes relapses were observed during one year in all patients.

Therefore, inclusion of interleukin-1ßinto complex therapy of severe forms of herpes recidivicus, allows to remove very effectively immune disturbances and to prevent occurrence of new infection relapses. Prophylactic prescription of interleukin-1 $\beta$ during remission period of herpes recidivicusakso demonstrated advisability of the therapy. On the background of therapy with interleukin- $1 \beta, 12$ patients had remission duration. So, in 10 patients prior to therapy with interleukin-1 $\beta$, remission duration was $1.5-2$ months, and after the therapy in 9 of them it increased to $10-11$ months. In 5 female patients, remission duration was $2-3$ months, after the treatment using interleukin-1 $\beta$ remission lasted more than 12 months. 3 patients during 2-year follow-up had no relapses at all. Anti-recurrent effect of interleukin-1 $\beta$ is related with immune modulatory properties of the preparation, consisting of increased content of ripe lymphocytes and active lymphocytes (CD25+), as well as increased values of spontaneous and induced LPS NST-test.

During the moderate and severe HSV-infection, manifested T-cell link depression is noted, expressed in reliable decrease of relative CD3+, CD4+ lymphocytes content, while relative content of B-lymphocytes and natural killers of CD16+ cells and lymphocytes, expressing adhesion receptors $\mathrm{CD} 11 \mathrm{~b}+$ and early and late activation markers, is compensatory increased.

In patients with moderate and severe HSVinfection on systemic level: catching ability of microphages is inhibited, and digesting ability is satisfactory. On the local level, conversely, phagocytic activity of neutrophils is high, at that, metabolic activity inhibition to a greater extent is more typical for cervical secretion microphages.

In patients with moderate and severe HSVinfection, manifested decrease of cytokines level is observed: FNO $\alpha$, IL-1 $\beta$, INF $\gamma$, IL-4.

Prescription of interleukin-1ßin $500 \mathrm{ng}$ dose every other day in the course of 5 injection in combination with acyclovir $200 \mathrm{mg} 5$ times a day during the moderate and severe HSVinfection leads to regression of herpes clinical 
manifestations at treatment days 2 to 3 , to remission duration increased more than 2-fold (more than 12 months), normalization of initially decreased CD3+, CD4+ lymphocytes content with preservation of high levels of CD8+, CD16+, CD56+, CD20+ lymphocytes and activation markers, values of spontaneous and induced LPS NST-test.

Prophylactic application of interleukin-1 $\beta$ in monotherapy during the remission of moderate and severe HSV-infection leads to aborted course of next relapse, increased remission duration of more than $12-24$ months, increased ripe lymphocytes and active lymphocytes (CD25+) content, as well as increased values of spontaneous and induced LPS NST-test.

\section{References}

Aspetov DR. Development of immunobiological products for the diagnosis, prevention and treatment of chronic viral infections (herpes, hepatitis $\mathrm{B}$ and $\mathrm{C}$ ). Diss....doctor med.sciences. Almaty, 2006.

Grenebyuk V.N. Recurrent genital herpes. Clinic, immunoreactions properties, treatment. Diss.doctor med.sciences. M, 1993; 48 p.

Isakov VA, Selkov SA, Moshetova LK, Chernakova GM. Current therapy of herpes virus infections: a guide for doctors. - SPb., 2004; 168 p.

Jansen MA, van den Heuvel D, Bouthoorn SH, Jaddoe VW, Hooijkaas H, Raat H, Fraaij
PL, van Zelm MC, Moll HA. Determinants of Ethnic Differences in Cytomegalovirus, Epstein-Bar Virus, and Herpes Simplex Virus Type 1 Seroprevalence in Childhood. J Pediatr. 2015; 0022-3476(15)01349-9.

Kinghorn G.R. Epidemiology of genital herpes/ J. Int. Med. Res. 1994; 22:14A-23A.

Kozlova V.I., and Puchner A.F. Viral, chlamydial and mycoplasmial genital diseases. M, 2003; 332.

Kurmanova A.M., Mamedaliyeva N.M., Kurmanova G.M., Dzoz L.S., Sartayeva K.A., Tohtakulinova G.K. Evaluating the effectiveness of inosinepranobex and interleikin 2 with genital viral infections // Int. J on immunorehabilitation. 2014; 2:122.

Marchenko L.A. Modern clinical aspects of the epidemiology of genital herpes. Diseases of the cervix, vagina and vulva (Clinical lectures) / Ed. Prof. V.N. Prilepskaya - M: Medpress, 2000; 220-228.

Nygmanova GT. The domestic immunobiological preparations in the treatment and prevention of recurrent labial and genital herpes // Dis.... cand med sciences. Almaty, 2005; 103.

Simbirtsev AS Medical preparations on the basis of family proteins interleukin-1 // Handbook of immunotherapy for practitioners/Ed. AS Simbirtsev. Publishing house "Dialog", 2002:152157.

Zudin B.I., and Kissina V.I. Genital herpes. STD. 1995; 3: 73-75.

\section{How to cite this article:}

Smailova, L.K., A.M. Kurmanova, G.M. Kurmanova, R.R. Razumova and Eszhanova, A.A. 2018. Immuno Correction of Genital Herpes of Viral Infection Moderate and Severe Course Betaleikin. Int.J.Curr.Microbiol.App.Sci. 7(02): 2506-2514. doi: https://doi.org/10.20546/ijcmas.2018.702.305 